\title{
Agenesis of hepatic lobes in a dog
}

\author{
Yasuhisa Oishi ${ }^{1}$, Kenji Tani $^{1, *}$, Kazuhito Itamoto ${ }^{2}$, Tomoya Haraguchi ${ }^{2}$ and Yasuho Taura $^{1}$ \\ ${ }^{1}$ Department of Veterinary Surgery, Joint Faculty of Veterinary Medicine, Yamaguchi University, 1677-1, Yoshida, \\ Yamaguchi 753-8511, Japan \\ ${ }^{2}$ Department of Animal Medical Center, Joint Faculty of Veterinary Medicine, Yamaguchi University, 1677-1, \\ Yoshida, Yamaguchi 753-8511, Japan
}

\begin{abstract}
Agenesis of a hepatic lobe is an extremely rare congenital anomaly and only one dog have been reported in veterinary literature. We encountered a dog with this anomaly diagnosed by Computed tomography (CT) and portography. A two-year-old, 6.9-kg female Shih tzu dog was presented with vomiting and anorexia. The dog had no history of abdominal surgery or trauma. Biochemical analysis showed elevated plasmatic liver enzymes. CT revealed the absence of the liver parenchyma and vascular system of the left lobe, quadrate lobe and papillary process of the caudate lobe. A portosystemic shunt was also observed. The liver parenchyma and vascular system of these lobes were not detected under digital subtraction angiography during laparotomy. Furthermore, the liver parenchyma and vascular system of these lobes were not detected even when the remaining liver volume increased two months after treating the shunt vessel. CT proved itself a good option for antemortally diagnosis of hepatic agenesis in a dog.
\end{abstract}

Keywords: Computed tomography, Dog, Hepatic lobe agenesis, Liver, Portosystemic shunts.

\section{Introduction}

Agenesis of a hepatic lobe is extremely rare in dogs. Humans with agenesis of a hepatic lobe usually show no clinical symptoms (Inoue et al., 1997). Therefore, detecting this type of agenesis is difficult based on symptoms, and rather, it is an incidental finding revealed by ultrasonography or computed tomography (CT) (Nacif et al., 2012). Agenesis of the hepatic lobe is commonly associated with other anatomical alterations, such as hypertrophy of other liver segments, colonic interposition between the liver and diaphragm, portal hypertension or an anomalously positioned gallbladder (Radin et al., 1987; Iannelli et al., 2005).

Here we report a case of agenesis of hepatic lobes in a dog with portosystemic shunt (PSS).

\section{Case Details}

A two-year-old female Shih tzu dog, weighting $6.9 \mathrm{~kg}$, presented for investigation of a two-week history of anorexia and vomiting. The dog had no history of abdominal surgery or trauma. No significant abnormalities were observed on physical examination. The body condition score of the dog was 5/9. Hematological examination revealed a decreased mean corpuscular volume but no other remarkable findings. Biochemical analysis showed increased aspartate aminotransferase $(179 \mathrm{U} / l)$, alanine aminotransferase (1001 U/l), alkaline phosphatase (9408 U/l), gammaglutamyl transpeptidase $(56 \mathrm{U} / l)$, ammonia $(92 \mu \mathrm{g} / \mathrm{d} l)$ and C-reactive protein $(1.2 \mathrm{mg} / \mathrm{d} l)$, whereas blood urea nitrogen $(5.5 \mathrm{mg} / \mathrm{d} l)$ was decreased. Prothrombin (14.4 $\mathrm{s}$; reference range, 4.68-7.04 s) and activated partial thromboplastin (27.1 s; reference range, 11.12-15.93 s) times were delayed, whereas antithrombin III (65.3\%; reference range, $>95 \%$ ) was decreased. Post operative biochemical data are listed in Table 1.

An abdominal radiograph revealed that gastric silhouette was parallel with the diaphragm outline (Fig. 1). Ultrasonography revealed an enlarged gallbladder and bile duct and absence of the left lobe of the liver.

Table 1. Biochemical results of a two-year-old female Shih tzu dog presenting hepatic lobe agenesis.

\begin{tabular}{ccccccc}
\hline Para & Pre & 3days & 7days & 14days & 68 days & Reference \\
\hline AST & 179 & 84 & 143 & 42 & 187 & $\begin{array}{c}9.2-29.2 \\
\text { IU/l }\end{array}$ \\
ALT & 1001 & 360 & 251 & 288 & 1240 & $\begin{array}{c}17-78 \\
\text { IU/1 }\end{array}$ \\
\hline ALP & 9408 & 8020 & 7593 & 6794 & 2634 & $\begin{array}{c}47-254 \\
\mathrm{IU} / 1\end{array}$ \\
\hline GGT & 56 & 31 & 47 & 52 & 25 & $\begin{array}{c}5-14 \\
\mathrm{IU} / \mathrm{l}\end{array}$ \\
\hline NH3 & 92 & 76 & 38 & 41 & 44 & $\begin{array}{c}16-75 \\
\mu \mathrm{g} / \mathrm{dl}\end{array}$ \\
\hline BUN & 5.5 & 3 & 2.8 & 4.5 & 10.8 & $\begin{array}{c}9.2-29.2 \\
\mathrm{mg} / \mathrm{dl}\end{array}$ \\
\hline Cre & 0.3 & 0.3 & 0.3 & 0.5 & 0.3 & $\begin{array}{c}0.4-1.4 \\
\mathrm{mg} / \mathrm{dl} \\
<1.0 \\
\mathrm{mg} / \mathrm{dl}\end{array}$ \\
\hline CRP & 1.2 & 3.55 & 1.1 & 0.6 & 0.3 & \\
\hline
\end{tabular}

(Para): Parameter; (AST): aspartate transaminase; (ALT): alanine transaminase; (ALP): alkaline phosphatase; (GGT): gamma-glutamyl transferase; (NH3): ammonia; (BUN): blood urea nitrogen; (Cre): creatinine; (CRP): c-reactive protein. 

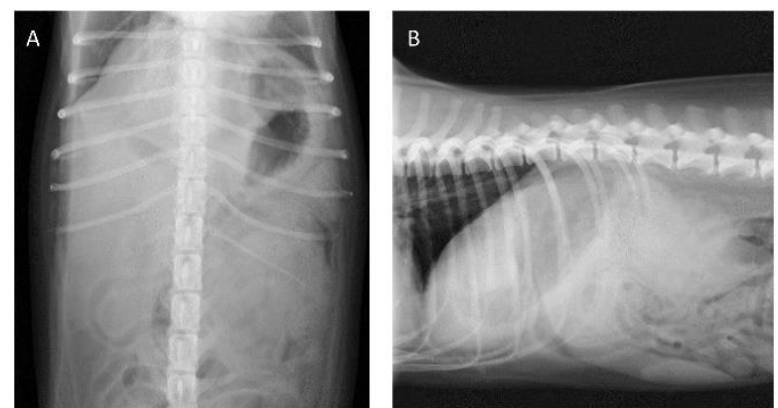

Fig. 1. Abdominal radiographs of a 2-year-old female Shih tzu dog. (A): Ventral-dorsal radiograph reveals that gastric silhouette is displaced toward the left abdominal wall. (B): lateral radiograph reveals gastric silhouette parallel with the diaphragm outline.

CT scanning was performed under general anesthesia. A survey helical CT scan of the abdomen revealed an enlarged gallbladder and absence of the left lobe, quadrate lobe and papillary process of the caudate liver lobe. The dog received $2 \mathrm{ml} / \mathrm{kg}$ of iopamidol with an iodine concentration of $370 \mathrm{mg} / \mathrm{ml}$ (Oiparomin 370; Fuji Pharmaceutical Co., Tokyo, Japan) as an intravenous contrast agent for vascular imaging. The contrast medium was injected with an automatic power injector (A-60; Nemoto Kyorindo Co., Ltd., Tokyo, Japan) at $1-2 \mathrm{ml} / \mathrm{s}$, and arterial phase imaging started 15 $s$ after contrast injection.

Portal phase imaging was started $50 \mathrm{~s}$ and equilibrium phase imaging was started $150 \mathrm{~s}$ after beginning of injection. No arteries of the left lobe, quadrate lobe and papillary process of the caudate lobe were observed during the arterial phase. No portal and hepatic veins of these lobes were observed during the portal and equilibrium phases (Figs. 2 and 3 ).

In addition, a shunt vessel was detected between the caudal vena cava and left gastric vein. The threedimensional reconstruction of the hepatic vasculature was performed using volume measurement software (Ziostation2; Amin Stock Co.). Preoperative liver volume was $127 \mathrm{cc}(18.4 \mathrm{cc} / \mathrm{kg})$, according to the software. The dog underwent surgical treatment the next day. The left lobe, quadrate lobe and papillary process of the caudate lobe were not observed under ventral midline laparotomy. The shunt vessel was visualized following the injection of contrast medium (Optiray 350; Covidien Co., Dublin, Ireland) during digital subtraction angiography (DSA).

Portal vein pressure was $10.5 \mathrm{~mm} \mathrm{Hg}$ before treatment and $15 \mathrm{~mm} \mathrm{Hg}$ after ligation with cellophane. No portal vein branches of the left lobe, quadrate lobe and papillary process of the caudate lobe were visualized under DSA guidance, even after temporary ligation (Fig. 4A). A biopsy sample was obtained from the right hepatic lobe.
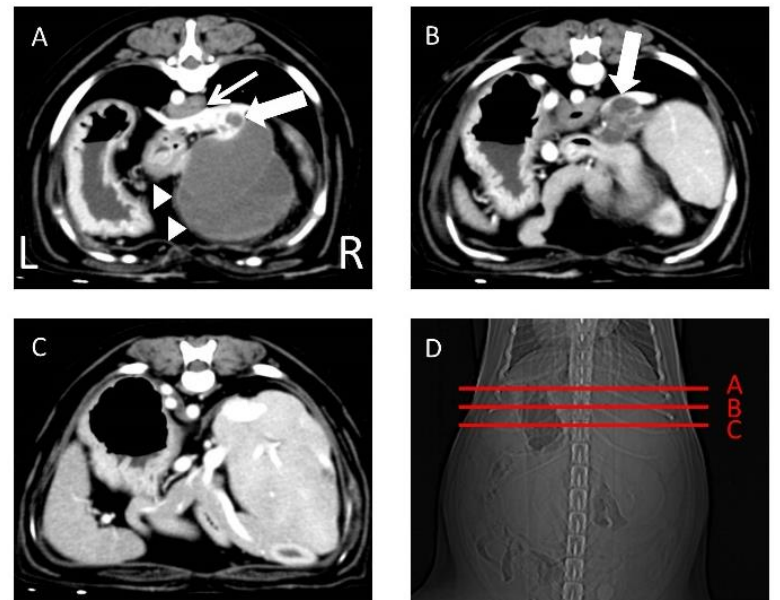

Fig. 2. Transverse computed tomography images of a twoyear-old female Shih tzu dog before surgical treatment. (A): Dorsal-ventral abdominal radiograph. Three lines reveal each transverse section level. Transverse computed tomography images at levels of (B) the tenth, (C) the eleventh and (D) the twelfth thoracic vertebrae in the portal phase revealed an enlarged bile duct (large arrow: blue) and gallbladder (arrowhead: red) and a shunt vessel (small arrow: yellow) between the caudal vena cava and left gastric vein. No left liver parenchyma was observed at any transverse section levels.
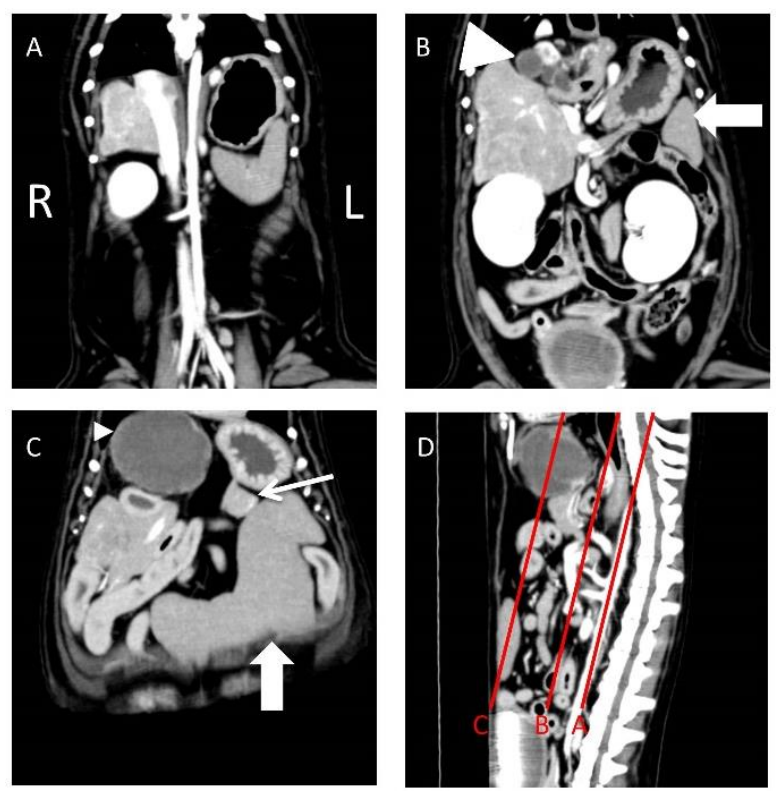

Fig. 3. Computed tomography multiplanar reconstruction (MPR) images of a two-year-old female Shih tzu dog before surgical treatment. (A): Sagittal MPR image. Three lines reveal each dorsal section level. (B-D): Dorsal MPR images reveal that the greater part of the left diaphragm is attached to the stomach and the remaining liver exists only on the right side of the gallbladder. Large arrow (blue) shows spleen, small arrow (yellow) shows pancreas, large arrowhead (red) showed bile duct and small arrowhead (red) shows the gallbladder. No left liver parenchyma was observed at any dorsal section levels. 

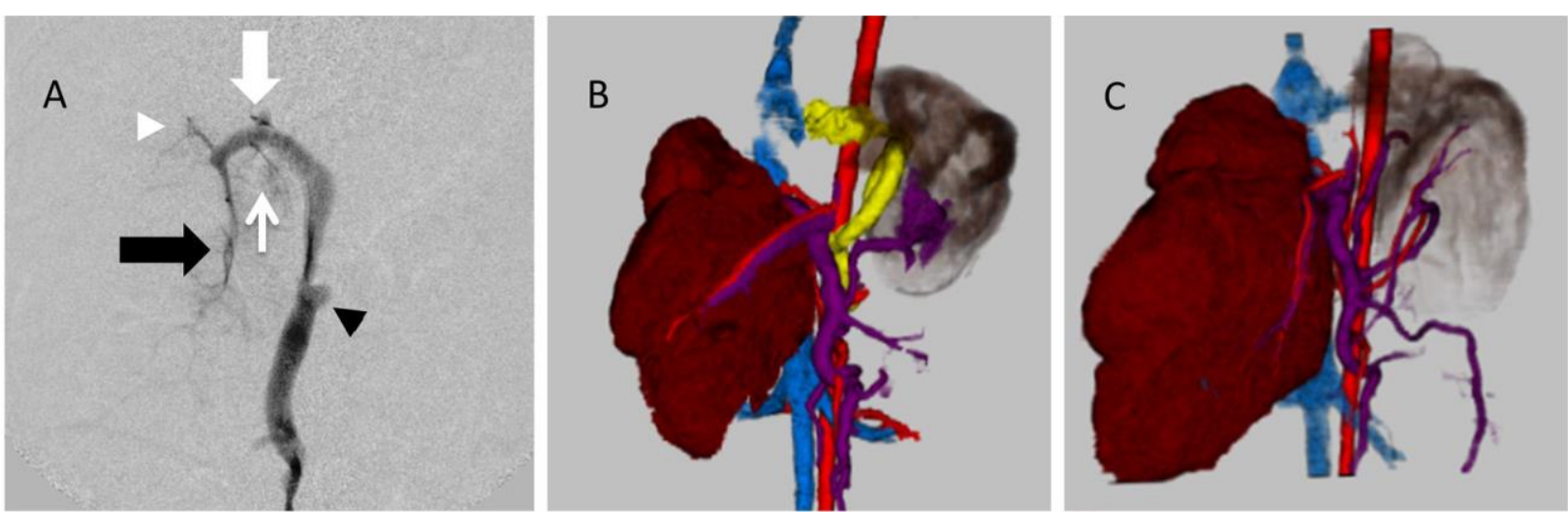

Fig. 4. Portography and three-dimensional computed tomography (3D-CT) images of a two-year-old female Shih tzu dog. (A): Portography after temporary occlusion in the remaining liver. Portal veins of the right medial hepatic lobe (large black arrow), right lateral hepatic lobe (white arrowhead) and caudate process of the caudate lobe (white arrow) were visualized, but no other vessels were observed. Large white arrow shows a gastroduodenal vein. (B): Preoperative 3D-CT image. Preoperative liver volume was $127 \mathrm{cc}(18.4 \mathrm{cc} / \mathrm{kg})$. Yellow: abnormal shunt vessel, red: artery, blue: vena cava and purple: portal vein. (C): Postoperative 3D-CT image. Postoperative liver volume was $187 \mathrm{cc}(27.1 \mathrm{cc} / \mathrm{kg})$. Gastroduodenal vessels were moved to left after surgery due to liver volume increasing.

Postoperative CT scanning revealed no blood flow to the shunt vessel and increased portal perfusion compared to those before surgery. Liver volume two months after surgery was $187 \mathrm{cc}(27.1 \mathrm{cc} / \mathrm{kg})$, which showed an increase of 47.2\% (Figs. 4B and 4C).

Liver parenchyma and hepatic vascular system of the left, quadrate and papillary process of the caudate lobe were not detected even after liver volume increase. Histopathological findings showed remodeling of the lobular structure, scattered hepatocytic vacuolization, arteriolar proliferation, biliary proliferation and fibrosis. Both aerobic and anaerobic cultures of bile showed no growth. Elevated liver enzymes decreased gradually but not to within normal range two weeks after surgery. Ammonia ( $44 \mu \mathrm{g} / \mathrm{d} l$; reference range, $16-$ $75 \mu \mathrm{g} / \mathrm{d} l)$ decreased to within the normal range. In addition, clinical symptoms improved gradually after surgery.

Discussion
In the present case, agenesis of hepatic lobes was diagnosed antemortally by CT. The causes of agenesis are not well known but the supposed etiology is dysgenesis of the hepatic primordium during development (Saigusa et al., 2001). A patient with agenesis of the hepatic lobe usually does not show any clinical symptoms.

Therefore, only a few patients have been diagnosed with agenesis, and the diagnosis in humans is generally incidental during imaging (Ormeci et al., 2016; Matsushita et al., 2017).

Recently, in veterinary literature, liver malformation due to diaphragmatic hernia was reported in puppies whose mother was under doxycycline, prednisone and tramadol administration (Kaplan et al., 2018). However, no similar information was observed in this case. One of 30 dogs with ductal plate malformations
(DPMs) had agenesis of a liver lobe and two of those dogs had a lobar atrophy (Pillai et al., 2016). Ductal plate malformations represent developmental biliary disorders and is characterized by expansion of portal areas with dense fibrous connective tissue, increased small clustered biliary profiles, portal-to-portal fibrosis, small caliber bile ducts intersecting directly hepatocytes through the limiting plate, increased arteriolar profiles with muscular walls, inconspicuous portal vein profiles and minimal portal inflammatory infiltrates with a wide phenotypic spectrum (Pillai et al., 2016). In this case, arteriolar proliferation and biliary proliferation with fibrosis were recognized although special stains were not performed. Thus, this case may be one of a wide phenotypic spectrum of DPMs.

Portosystemic shunts (PSS) are anomalous vessels that join the portal and systemic venous circulation and are classified as congenital or acquired (Stieger et al., 2007; Doran et al., 2008). Congenital extrahepatic PSS usually have a single, anomalous shunt vessel and is rarely associated with hepatic disease or portal hypertension (Doran et al., 2008). Acquired PSS develop secondary to portal hypertension (Buob et al., 2011). In the present case, the dog had a single PSS vessel. Contrast-enhanced CT or DSA images indicated PSS to be congenital in this dog.

However, histopathologic findings showed hepatitis with fibrosis which may be associated with acquired conditions. DPMs can be a cause of liver disease to induce acquired PSS due to portal hypertension. A congenital PSS can often be resolved with surgery whereas in acquired PSS it is ineffective. Although the remaining liver volume increased two months after surgery, long term follow-up is needed to access surgical effectiveness. 
Agenesis of the right lobe of the liver in humans is defined as the absence of liver tissue on the right side, with preservation of the middle hepatic vein in the absence of previous disease or surgery (Nacif et al., 2012). Although liver morphology differs between humans and dogs, the absence of liver tissue and vessels in the present case is similar to human agenesis of the hepatic lobe. Determining whether such an absence is congenital or acquired in any particular case can be difficult because severe atrophy can mimic agenesis, unless it is diagnosed in the first days/ weeks of life (Kakitsubata et al., 1991).

In the present case, single PSS was also observed. Although PSS can cause liver hypoplasia, there should be hepatic vessels and liver tissue in a dog with PSS (Stieger et al., 2007; Doran et al., 2008). However, in the present case, the liver tissue and hepatic vessels of the left lobe, quadrate lobe and papillary process of the caudate lobe were not observed in CT scanning and laparotomy. In addition, these lobes were not detected even when the remaining liver volume increased two months after the surgery. These observations suggest a congenital anomaly of hepatic parenchyma in our patient.

Agenesis of hepatic lobe is a rare finding, and appears to be overlooked since the condition is asymptomatic. The present case demonstrates the importance of knowing the normal hepatic vascular anatomy (Oishi et al., 2015) and the use of CT as the main diagnostic tool for identifying agenesis of hepatic lobe in the dog.

\section{Conclusion}

The present case demonstrates that CT is a good option for diagnosing agenesis of the hepatic lobe antemortally.

\section{Acknowledgements}

The authors are thankful to the staff of Animal Medical Center in Yamaguchi University for their animal support. This case report was supported, in part, by JSPS KAKENHI Grant Number 25234567.

\section{Conflict of interest}

The authors declare that there is no conflict of interest.

\section{References}

Buob, S., Johnston, A.N. and Webster, C.R. 2011. Portal hypertension: pathophysiology, diagnosis, and treatment. J. Vet. Intern. Med. 25, 169-186.

Doran, I.P., Barr, F.J., Hotston Moore, A., Knowles, T.G. and Holt, P.E. 2008. Liver size, bodyweight, and tolerance to acute complete occlusion of congenital extrahepatic portosystemic shunts in dogs. Vet. Surg. 37, 656-662.

Iannelli, A., Facchiano, E., Fabiani, P., Sejor, E., Bernard, J.L., Niezar, E. and Gugenheim, J. 2005. Agenesis of the right liver: a difficult laparoscopic cholecystectomy. J. Laparoendosc. Adv. Surg. Tech. A. 15, 166-169.

Inoue, T., Ito, Y., Matsuzaki, Y., Okauchi, Y., Kondo, H., Horiuchi, N., Nakao, K. and Iwata, M. 1997. Hypogenesis of right hepatic lobe accompanied by portal hypertension: case report and review of 31 Japanese cases. J. Gastroenterol. 32, 836-842.

Kakitsubata, Y., Nakamura, R., Mitsuo, H., Suzuki, Y., Kakitsubata, S. and Watanabe, K. 1991. Absence of the left lobe of the liver: US and CT appearance. Gastrointest. Radiol. 16, 323-325.

Kaplan, J.L., Gunther-Harrington, C.T., Sutton, J.S. and Stern, J.A. 2018. Multiple midline defects identified in a litter of golden retrievers following gestational administration of prednisone and doxycycline: a case series. BMC Vet. Res. 14, 86. Doi: https://doi.org/10.1186/s12917-018-1419-y.

Matsushita, K., Gotoh, K., Eguchi, H., Iwagami, Y., Yamada, D., Asaoka, T., Noda, T., Wada, H., Kawamoto, K., Doki, Y. and Mori, M. 2017. Agenesis of the left hepatic lobe undergoing laparoscopic hepatectomy for hepatocellular carcinoma: a case report. Surg. Case Rep. 3, 50. Doi: https://doi.org/10.1186/s40792-017-0325-z.

Nacif, L.S., Buscariolli, Yo.S., D'Albuquerque, L.A. and Andraus, W. 2012. Agenesis of the right hepatic lobe. Case Rep. Med. 415742. Doi: http://dx.doi.org/10.1155/2012/415742.

Oishi, Y., Tani, K., Nakazawa, H., Itamoto, K., Haraguchi, T. and Taura, Y. 2015. Anatomical evaluation of hepatic vascular system in healthy beagles using X-ray contrast computed tomography. J. Vet. Med. Sci. 77, 925-929.

Ormeci, T., Erdogan, S.T., Ormeci, A. and Aygun, C. 2016. A rare congenital liver anomaly: Hypoplasia of left hepatic lobe. J. Pak. Med. Assoc. 66(12), 1662-1664.

Pillai, S., Center, S.A., McDonough, S.P., Demarco, J., Pintar, J., Henderson, A.K., Cooper, J., Bolton, T., Sharpe, K., Hill, S., Benedict, A.G. and Haviland, R. 2016. Ductal Plate Malformation in the Liver of Boxer Dogs: Clinical and Histological Features. Vet. Pathol. 53, 602-613.

Radin, D.R., Colletti, P.M., Ralls, P.W., Boswell, W.D. and Halls, J.M. 1987. Agenesis of the right lobe of the liver. Radiology 164, 639-642.

Saigusa, K., Aoki, Y. and Horiguchi, M. 2001. Hypoplasia of the left lobe of the liver. Surg. Radiol. Anat. 23, 345-347.

Stieger, S.M., Zwingenberger, A., Pollard, R.E., Kyles, A.E. and Wisner, E.R. 2007. Hepatic volume estimation using quantitative computed tomography in dogs with portosystemic shunts. Vet. Radiol. Ultrasound 48, 409-413. 